\title{
Minigene Analysis of the c.743 + 40A > G Intronic Variant in the CFTR Gene
}

\author{
Ayman El-Seedy"\#, Raed Farhat1, Marie-Claude Pasquet1,2, Alain Kitzis ${ }^{1,2}$, \\ Véronique Ladeveze ${ }^{1^{*}}$ \\ ${ }^{1}$ Génétique Moléculaire de Maladies Rares, Pôle Biologie Santé, Université de Poitiers, Poitiers, France \\ ${ }^{2}$ Centre Hospitalier Universitaire (CHU) de Poitiers, Poitiers, France \\ Email: veronique.ladeveze@univ-poitiers.fr
}

Received 23 April 2014; revised 26 May 2014; accepted 3 June 2014

Copyright (C) 2014 by authors and Scientific Research Publishing Inc.

This work is licensed under the Creative Commons Attribution International License (CC BY).

http://creativecommons.org/licenses/by/4.0/

(c) (i) Open Access

\begin{abstract}
Background: Since the identification of the cystic fibrosis transmembrane regulator (CFTR) gene in 1989, many polymorphisms have been identified in cystic fibrosis (CF) or CFTR-related disorders (CFTR-RDs) patients and still remain to be characterized at the molecular level. These polymorphisms are difficult to classify as pathogenic or non-disease causing because the polymorphisms are either located in the coding region, but are synonymous, or are found in the intronic regions. Here we investigated the potential impact of the c.743 $+40 \mathrm{~A}>\mathrm{G}$ polymorphism within CFTR intron 6 on the alternative splicing. Indeed, this variant has been observed frequently in our examined patients. Moreover, a family carrying this variant exhibited $C F T R$-RD phenotype. Methods: By denaturing high pressure liquid phase chromatography (DHPLC) and sequencing, thirty of 293 subjects French origin carried the c.743 + 40A $>$ G variant. Of these, 16 patients were affected by CF or CFTR-RD. Wild-type sequences and mutant CFTR intron 6 and its boundaries were inserted into the pTBNdeI hybride minigene and expressed in three different cell lines. After RT-PCR analysis of mRNA using specific primers, sequences of the minigene transcripts were obtained. Results: No aberrant splicing was detected with minigene carrying c.743 + 40A $>$ G variant in all transfected cell lines. However, an alternative splicing in the positive control was detected with a minigene carrying the c.1392G $>\mathrm{T}+1 \mathrm{G}>\mathrm{T}$ mutation: 5 nucleotides were deleted from mRNA sequences, indicating that used cell lines are appropriate for studying the splicing. Conclusion: Transient transfections of a minigene containing the c.743 $+40 \mathrm{~A}>\mathrm{G}$ polymorphism showed no splicing errors, and thus this intronic alteration was finally classified as nonpathogenic. As it is always associated with c.2562T > G and c.4389G > A, or TG12-7T polymorphisms, further experiments are needed to determine the role of these complex alleles in disease pathogenesis.
\end{abstract}

\footnotetext{
${ }^{\#}$ Present address: Department of Genetics, Alexandria University, Aflaton St. 21545-EL-Shatby, Alexandria, Egypt.
} ${ }^{*}$ Corresponding author. 


\section{Keywords}

\section{Cystic Fibrosis, Polymorphism, Complex Allele, Hybrid Minigene, Splicing}

\section{Introduction}

Cystic fibrosis (CF) is an autosomal recessive genetic disorder caused by a variety of sequence alterations in the CFTR (Cystic Fibrosis Transmembrane regulator conductance) gene. Up to now, more than 1960 mutations and polymorphisms have been reported to the Cystic Fibrosis Mutation Database [1]. Genomic surveys indicate that polymorphisms affecting transcription and mRNA processing, including splicing and turnover, may account for the main share of genetic factors in human phenotypic variability; however, most of these polymorphisms remain yet to be understood [2]. After complete sequence analysis of the CFTR gene in various laboratories, many polymorphisms have been identified. Molecular and cellular studies have also uncovered a large number of putative mutations or polymorphisms which showed phenotypic variability. For this reason, further studies are still needed to determine their eventually pathological effects. So, the comprehensive study will help in giving an accurate genetic counselling and prognosis for patients or individuals.

In this respect, it is now clear that single nucleotide polymorphisms may also strongly influence gene expression at the splicing level [3]. Moreover, functional polymorphisms in the CFTR gene can alter the expression of the CFTR protein [3]. Previous studies have suggested that the most frequent polymorphism in CFTR gene, the c.1408A > G in exon 11, (p.Met470 Val) plays a role in modulating CFTR protein at both the transcriptional and translational levels. It was reported that the Met470 CFTR was associated with a 1.7-fold of the Val470 CFTR function [4]. Similarly, the cDNA single-nucleotide polymorphisms c.2562T > G (exon 15) and c.4389G > A (exon 27) may affect RNA splicing by changing regulatory-sequence motifs of exonic splice enhancers, leading to increased lower amounts of normal transcripts [5]. In addition, the polythymidine variants in intron 9 are associated with the efficient usage of the intron 9 splicing acceptor site, and induce exon 10 skipping. The $5 \mathrm{~T}$ results in approximately $90 \%$ of exon 10 skipping, leading to a non-functional CFTR, thereby are considered as a disease mutation with incomplete penetrance [6].

More recently, the c.743 $+40 \mathrm{~A}>\mathrm{G}$ polymorphism in intron 6 is found as a frequent polymorphism after an extensive molecular genetic investigation at the CFTR gene in French CFTR-related disorders (CFTR-RDs) patients. It was classified as non-CF-causing allele on the basis of its frequency [7]. This polymorphism has sporadically been observed as uncommon in Uruguayan [8]; Slovac [9], and Algerian patients [10] but relatively frequent $(2.5 \%-4.0 \%)$ in Spanish patients [11].

Interestingly, we present here a family carrying the c.743 $+40 \mathrm{~A}>\mathrm{G}$ polymorphism in cis with c.2562G and c.4389T variants on the same allele in exons 15 and 27 respectively whereas each of them has no effect on CFTR. The father and his son had pancreatic inflammation with elevated sweat chloride levels in the absence of cystic fibrosis or any deleterious mutation. In order to investigate the eventual pathogenecity of the c.743 $+40 \mathrm{~A}>$ G polymorphism we examine its effect upon mRNA level in cellulo using cultured cell-line (HeLa, HT29, HEK293) for minigene expression, a highly sensitive tool used to regularly screen putative CFTR mutations in CF patients.

\section{Material and Methods}

\subsection{Ethics Statement}

The study was performed according to French legislation and the recommendations of the local ethics committee in accordance with the Declaration of Helsinki. Written consent to the genetic study was obtained from all patients before withdrawal of blood as well as their permission for publication.

\subsection{Mutation Nomenclature}

The recommendations of the Human Genome Variation Society (http://www.hgvs.org/mutnomen/) were followed for exon numbering and mutation names. The current recommendations suggest using +1 as the $\mathrm{A}$ of the 
initiation ATG codon in the the CFTR cDNA sequence (GenBank NM_000492.2) as the reference sequence.

\subsection{Analysis of c. $743+40 \mathrm{~A}>\mathrm{G}$ Variant in the General Population}

Between April 2007 and January 2010, 640 subjects French origin had been referred to our CF Center at the Hospital of Poitiers (CHU), for genetic counseling purposes were examined. All genomic DNA samples were extracted from peripheral blood cells using the QIAamp DNA Blood Mini kit (Qiagen) according to the supplier's protocol. Extensive CFTR gene analyses were performed in the patients using a combination of methods: 1) screening for frequent mutations using diverse commercial assays; 2) scanning of the 27 exons and their boundaries using denaturing gradient gel electrophoresis (DGGE) [12], or denaturing high pressure liquid phase chromatography (DHPLC) [13]. The screening of the c.743 + 40A > G variant has been performed by DHPLC analysis followed by automated DNA sequencing. PCR primers were: forward primer

5'-TCCTTTTACTTGCTTTCTTTCA-3' (HPCF-6aA) and reverse 5'-TATGCATAGAGCAGTCCTGGTT-3' (HPCF-6aB). The PCR conditions were as follows: denaturation at $95^{\circ} \mathrm{C}$ for $30 \mathrm{~s}$, annealing at $55^{\circ} \mathrm{C}$ for $30 \mathrm{~s}$ and extension at $74^{\circ} \mathrm{C}$ for $40 \mathrm{~s}$, for 32 cycles. DNA samples of cases were analysed by previously reported methods. All 27 exons of CFTR were amplified by PCR followed by direct sequencing of all exons and exon-intron boundaries.

\subsection{Generation of the Minigene Constructs}

The construction and validation of the hybrid minigene used in this study has been described elsewhere [14]. Briefly, a 1666bp PCR fragment encompassing exon6 and exon7 and its flanking introns were amplified from normal and mutated human genomic DNA. After restriction enzyme digestion of used plasmid by NdeI restriction enzyme site, PCR products were inserted into pTBNdeI plasmid (kindly donated by F. Pagani). All hybrid minigene constructs were sequenced to verify the correct insertion of the wild-type and mutated DNA fragments with 2.3 $\alpha$ and BraRev primers (Table 1). For the construction of the positive control c.1392 + 1G > T, after insertion of the 483bp fragment containing exon 10 and its adjacent introns, site directed mutagenesis was performed on the minigene containing the wild-type fragment with forward primer

5'GATCCACTGGAGCAGGCAAGTTAGTTCTTTTGT'3 and reverse primer

5'CTTGCCTGCTCCAGTGGATCCAGCAACCGC'3. Presence of the desired mutation and absence of PCRartifacts were confirmed by sequencing.

\subsection{Transfection, RNA Isolation and RT-PCR Amplification}

Cells were grown in a DMEM medium with Glutamax-I (Life Technologies) amended with $10 \%$ fetal bovine serum (FBS,Gibco), and $100 \mathrm{unit} / \mathrm{ml}$ penicillin and $100 \mu \mathrm{g} / \mathrm{ml}$ streptomycin in a humidified incubator at $37^{\circ} \mathrm{C}$ in the presence of $5 \% \mathrm{CO}_{2}$. WT and CFTR mutants were transiently expressed in HeLa, HT 29 and HEK 293 cells following the use of LipofectAMINE 2000 (Invitrogen) in accordance with the manufacturer's instructions. At least three independent transfections were performed in these three different lines. cDNA synthesis was carried out at $37^{\circ} \mathrm{C}$ for 1 hour. The reaction was stopped by 2 minute incubation at $100^{\circ} \mathrm{C}$. RT-PCR was performed using primers designed for the exons of plasmid (Table 1). PCR was performed using a 9700 GeneAmp Thermo Cycler (Perkin Elmer) under the following cycling conditions: initial denaturation at $94^{\circ} \mathrm{C}$ for 2 minutes, followed by 30 cycles at: $94^{\circ} \mathrm{C}$ for 30 seconds, $58^{\circ} \mathrm{C}$ for 30 seconds, and $72^{\circ} \mathrm{C}$ for 30 seconds, followed by a final 5 minutes extension step at $72^{\circ} \mathrm{C}$. The reaction was verified by $1.5 \%$ agarose gel electrophoresis.

Table 1. Olignucleotides using in RT-PCR, and sequencing of inserted fragment.

\begin{tabular}{cc}
\hline Name & Sequence \\
\hline pTB2160Dir & 5'-TATTCAGATATTTATGTCTAGG-3' \\
pTB2270Rev & 5'-CCCATGTGAGATATCTAGG-3 \\
${\mathbf{2 . 3 \alpha ^ { * }}}^{\text {BraRev }^{*}}$ & 5'-CAACTTCAAGCTCCTAAGCCACTGC-3' \\
\hline
\end{tabular}

*Indicate the sequence of RT-PCR olignucleotides. 


\subsection{DNA Sequence Analysis}

Sequence analysis was performed using the ABI PRISM ${ }^{\mathrm{TM}}$ Big Dye Terminator ${ }^{\mathrm{TM}}$ Cycle Sequencing Ready Kit (Applied Biosystems) and analyzed on an ABI PRISM ${ }^{\mathrm{TM}} 3130$ automatic sequencer (Applied Biosystems) according to the manufacturer's protocols. The obtained sequences were compared with the published sequence database (available at http://www.genet.sickkids.on.ca/cftr/app).

\subsection{Splice-Site Predictions}

In order to identify the potential impact of the c.743 + 40A > G variant on splicing, in silico analyses were performed with mutant and reference sequences using four different programs: Neural Network SPLICE (NN Splice) 0.9 from the Berkeley Drosophila Genome Project (available at http://www.fruitfly.org/seq_tools/splice.html), NetGene2 (available at http://www.cbs.dtu.dk/service/NetGene2/), Human Splicing Finder (HSF)-Version 2.4 (available at http://www.umd.be/HSF/), and Putative Exonic Splicing (PESX) (available at http://cubweb.biology.columbia.edu/Pesx/).

\section{Results}

\subsection{Analysis of c.743 + 40A > G Variant in the General Population}

During the genetic characterization of the 640 enrolled subjects, 293 participants have completed the comprehensive examination of intron 6 . Of these, 30 were carried the c.743 + 40A > G polymorphism within CFTR intron 6.16 patients of them were initially screened on the basis of complete clinical symptoms and were diagnosed as having CF or CFTR-RD. Figure 1 shows the abnormal DHPLC profile of the target variant in these patients. There is a high degree of phenotypic variability associated with this polymorphism. The c.743 + 40A > G allele was found in patients presenting: suspected CF $(n=2)$, congenital bilateral absence of vas deference (CBVAD) (n $=1)$, other causes of infertility $(n=7)$, disseminated bronchiectasis $(n=2)$, pulmonary disease $(n=2)$, and hyperechogenecity $(\mathrm{n}=2)$. Detailed information on CFTR genotype and clinical data from selected CF or CFTR-RD patients for this study had been reported in Table 2. TG12-7T haplotype were found in 9 patients. Two cases with the TG12-5T were found in the CBAVD and infertility patients suggesting that the haplotype TG12-5T might be the most common disease-associated combination and impair the process of CBAVD.

Table 2. Characterization of CFTR mutations in 16 patients carried the c. $743+40 \mathrm{~A}>\mathrm{G}$ polymorphism.

\begin{tabular}{|c|c|c|c|c|}
\hline Patients & $\begin{array}{c}\text { Identified } \\
\text { Mutations }\end{array}$ & Other variations & Tn, TGm & Clinical symptoms \\
\hline 1 & c.2875delG & c. $2562>$ G, c. $4272 \mathrm{C}>\mathrm{T}$ & 7/7T, 10/12TG & Positive sweat test (Suspicion of CF?) \\
\hline 2 & - & с. $2562>$ G, с. $4272 \mathrm{C}>$ T, с. $4389 \mathrm{G}>\mathrm{A}$ & 7/7T, 10/11TG & Chronic diarrhea, asthma, pancreatitis \\
\hline 3 & - & с. $2562>$ G, с. $4272 \mathrm{C}>$ T, с. $1408 \mathrm{~A}>\mathrm{G}$ & 7/9T, 10/10TG & $\mathrm{DB}$ \\
\hline 4 & - & c. $2562>$ G, с. $4272 \mathrm{C}>$ T, с. $4389 \mathrm{G}>\mathrm{A}$ & 7/9T, 10/11TG & Hyperechogenecity \\
\hline 5 & - & c. $2562>$ G, c. $4389 G>A$ & 5/7T, 11/11TG & Infertility \\
\hline 6 & S1235R & c. $2562>$ G, c.4389G > A & 5/7T, 11/12TG & Infertility \\
\hline 7 & L206W & c.256 2> G, c.4389G > A & 7/7T, 10/11TG & Asthma \\
\hline 8 & D1152H, G85E & c. $2562>$ G, c. $4389 \mathrm{G}>\mathrm{A}$, c. $1408 \mathrm{~A}>\mathrm{G}$ & 5/7T, 11/12TG & CBAVD \\
\hline 9 & - & c. $2562>$ G, c. $4389 G>A$ & 7/7T, 10/12TG & Infertility \\
\hline 10 & - & c. $2562>$ G, c. $4389 G>A$ & 7/7T, 10/12TG & Infertility \\
\hline 11 & - & c. $2562>$ G, c. $4389 G>A$ & 7/7T, 10/12TG & Infertility \\
\hline 12 & - & c. $2562>$ G, c. $4389 G>A$ & 7/7T, 10/12TG & Infertility \\
\hline 13 & - & c. $2562>$ G, c. $4389 G>A$ & 7/7T, 10/12TG & Infertility \\
\hline 14 & - & c. $2562>$ G, с. $4389 \mathrm{G}>\mathrm{A}, 1408 \mathrm{~A}>\mathrm{G}$ & 7/7T, 10/12TG & Positive sweat test (Suspicion of CF?) \\
\hline 15 & - & с. $2562>$ G, с. $4389 \mathrm{G}>$ A, с. $1408 \mathrm{~A}>\mathrm{G}$ & 7/7T, 10/12TG & Hyperechogenicity \\
\hline 16 & - & с. $869+11 \mathrm{C}>$ T, с. $1408 \mathrm{~A}>\mathrm{G}, 2694 \mathrm{~T}>\mathrm{G}$ & 7/7T, 10/12TG & DB \\
\hline
\end{tabular}




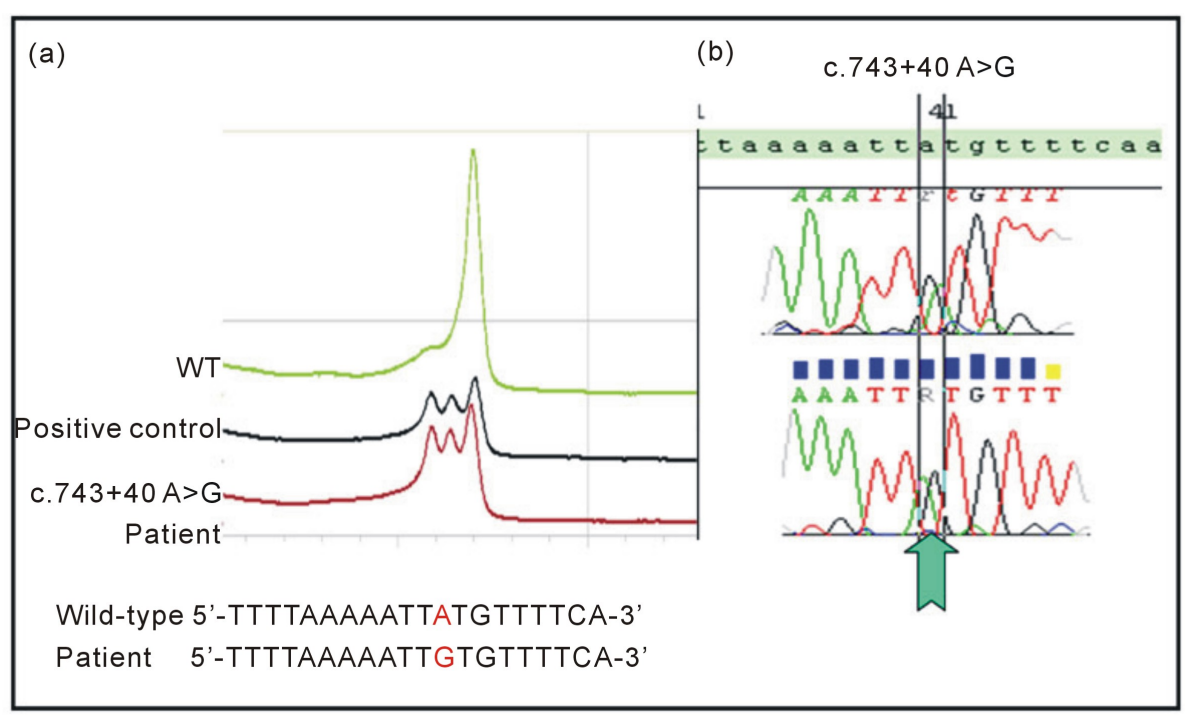

Figure 1. DHPLC chromatogram is shown the wild-type, and c.743 $+40 \mathrm{~A}>\mathrm{G}$ polymorphism compared to a positive control. (a) DHPLC profile of CFTR c.743 + 40A $>$ G polymorphism in contrast to the normal control. The top plot detects the wild-type peak. The bottom plot detects the mutant peak of $\mathrm{c} .743+40 \mathrm{~A}>\mathrm{G}$ polymorphism. The result shows that this patient is a carrier for this polymorphism. The middle plot detects the mutant peak of positive control. (b) Direct sequencing of Intron 6a confirmed the c.743 $+40 \mathrm{~A}>\mathrm{G}$ polymorphism (filled arrow).

\subsection{Family Data}

Here we present the main clinical feature of the family members. The family studied has the c.743 + 40A $>\mathrm{G}$ polymorphism, in association with c.2562T > G (exon 15), and the c.4389G > A (exon 27) polymorphisms in cis. The father has major health problems but not cystic fibrosis. This patient has a history of chronic diarrhea, but now can lead to a number of tools extremely important (40 to 50 per day), and while the specific genes for pancreatitis are normal (tested at the University Hospital of Brest). In addition, there is a suspicion of asthma since childhood related to nocturnal and wheezing dyspnea. The mother has one normal allele, and the other one c.2562T > G (exon 15) and c.4389G > A (exon 27). She shows no sign of deleterious symptoms. The couple has 2 children, and both carry the father's allele c. $743+40 \mathrm{~A}>\mathrm{G}$. The son in good health carries the WT allele of the mother, and the other one has the mutant allele from the mother and he has a pathology of type CFTR-RD, but less severe than his father.

\subsection{Splice-Site Predictions}

The effect of the predicted splicing variant (c.743 $+40 \mathrm{~A}>\mathrm{G}$ ) was first analyzed by computer-assisted splice-site prediction using the NNSplice, and NetGene2 programs. The programs recognized the wild-type as well as the mutant splice site without any appreciable score reduction. Conversely, HSFv2.4.1 program analysis revealed that the donor-site score ranged from 2.54 in the absence of polymorphism, to 3.37 when the substitution took place. Finally, PESX analysis showed minor changes in ESS distribution, whereas Rescue-ESE showed no change either in the wild-type or in the mutant sequence.

\subsection{CFTR Minigene Analysis of c.743 + 40A > G in Intron 6}

We investigated the effect of c.743 + 40A $>$ G variant using a hybrid minigene splicing approach. The primers used do not amplify eventual ectopically expressed CFTR and a 1666pb fragment specific was obtained (Figure 2(a) \& Figure 2(b)). After transfections, the 540 bp products are due to the correct splicing of intron 6 and its flanking exons, indicating that this polymorphism did not induce any aberrant splicing in the minigene assay (Figure 2(c)). Direct sequence showed a correct splicing (exon 6-exon 7). As we tested two times ( $24 \mathrm{~h}$ and $48 \mathrm{~h}$ ) our data suggested that the mutant RNA was correctly spliced and stably expressed (Figure 2(c)). Whereas the 


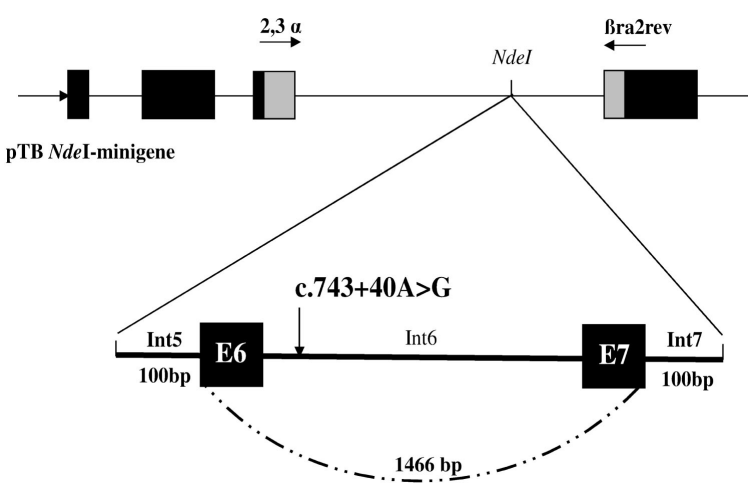

(a)

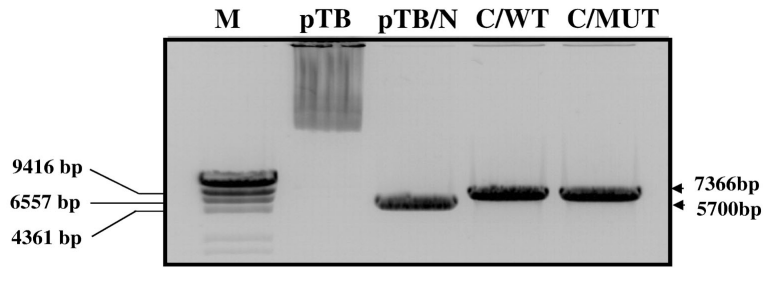

(b)

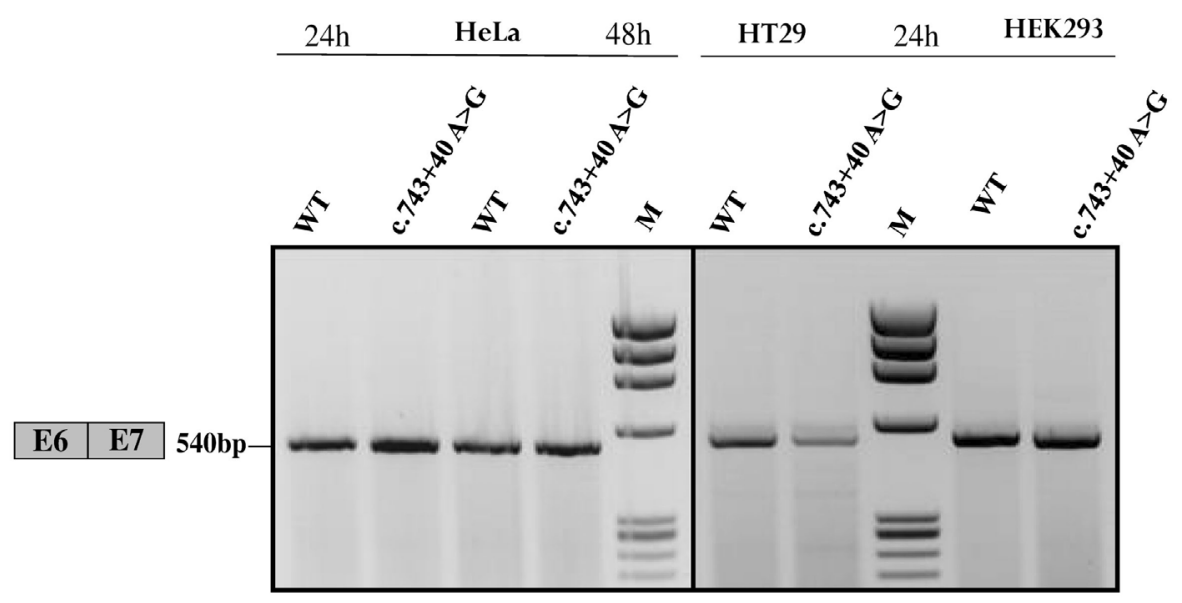

(c)

Figure 2. Effect of c.743 + 40A > G polymorphism on pre-mRNA splicing. (a) Schematic presentation of hybride minigene splicing construct used in transient transfection assay. Primers used in RT-PCR analysis are shown as black arrows (b) Linearization of different plasmids with NdeI shows the wild-type (pTB) minigene without NdeI cutting (lane 2), with NdeI cutting (pTB/N, lane 3), the wild-type (C/WT) minigene harboring the c.743 + 40A (lane 4), and the construct harboring the c.743 + $40 \mathrm{~A}>\mathrm{G}$ variant (C/MUT, lane 5). The used Marker is Lambda DNA/HindIII. (c) RT-PCR analysis of minigenes carrying the wild type sequence, WT, and c.743 + 40A $>$ G polymorphism, MUT, in intron 6a separated in a $1.5 \%$ agarose gel.

positive control minigenes which contained c.1392 + $1 \mathrm{G}>\mathrm{T}$ ( or $\mathrm{G}>\mathrm{C}$ ) mutations were composed of exon10 and its flanking introns of the CFTR gene (Figure 3(a)). DNA sequence of RT-PCR products generated with c. $1392+1 G>\mathrm{T}$ (or $\mathrm{G}>\mathrm{C}$ ) mutations showed a frameshift transcript, deleted of 5 nucleotides of exon 10 (Figure 3(b)), demonstrating that the cell lines used in this study were adapted to estimate splicing (Figure 3(c)). These experiments were repeated three times independently in three types of cells.

\section{Discussion}

A significant fraction (about 13\%) of CFTR mutations is classified as “splicing mutations”, but for almost $40 \%$ of these, their role in affecting the splicing is not yet defined. Splicing mutations act by directly disrupting splice sites or by creating new ones [15].

Studying the influence of polymorphisms on disease susceptibility in multiple ethnically and geographically distinct disease and control populations is important [16]. Over 200 polymorphisms have been identified within CFTR gene (www.genet.sickkids.on.ca/cftr). These do not cause CF, but may alter CFTR protein production and/or function. Such alterations may be clinically insignificant in individuals without additional CFTR mutations, but have an influence on disease phenotype in patients with co-existing mutations [17]. For example, the cDNA single-nucleotide polymorphisms c.2562T > G and c.4389G > A may affect pre-mRNA splicing by 


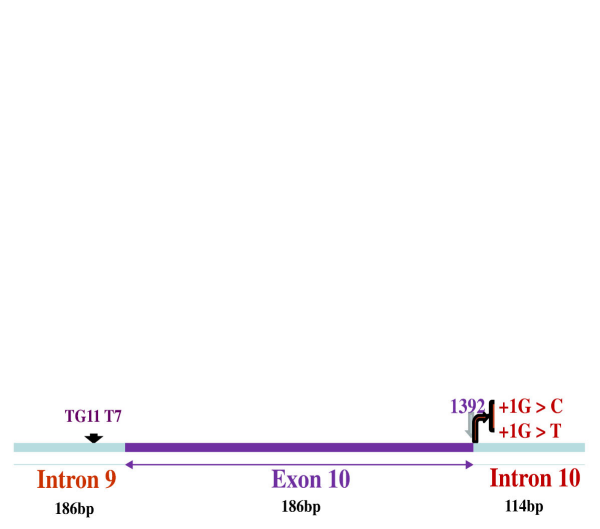

(a)

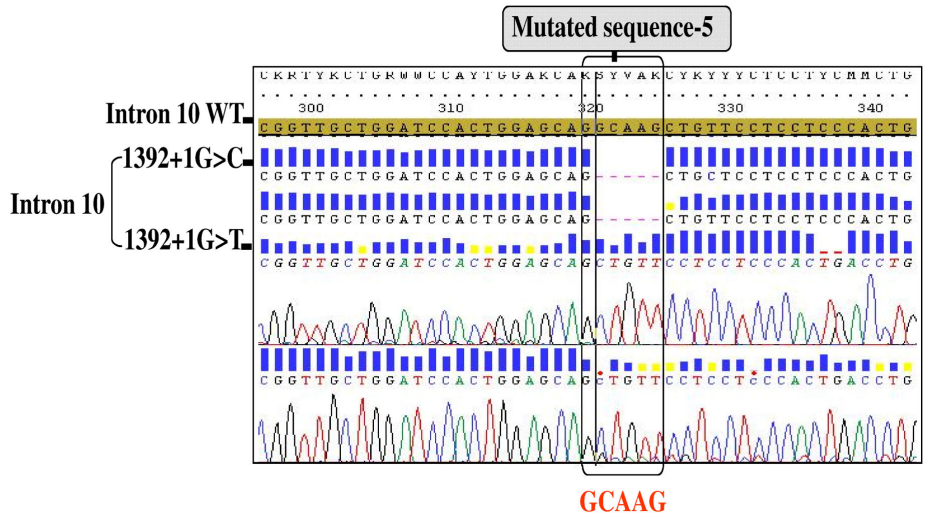

(b)

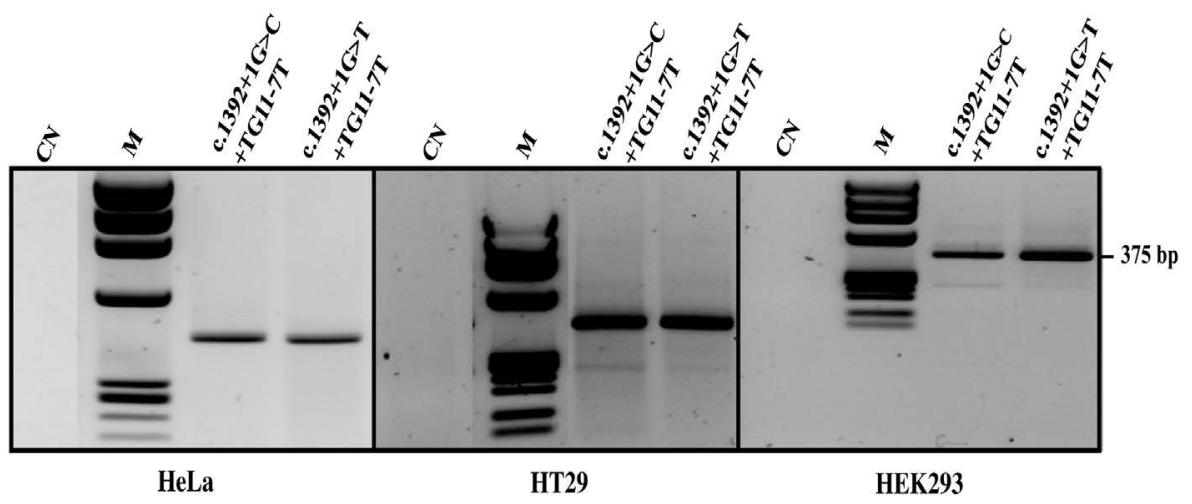

(c)

Figure 3. Analysis of CFTR mRNA by RT-PCR and direct sequence analysis of products generated with the positive control (a) Schematic diagram of a linear CFTR covering intron9-exon10-intron10 which use to bind to pTB minigene demonstrating the position of c.1392 + 1G > T (or G > C) mutations and the TG11-T7 polymorphism (b) DNA sequence of RT-PCR product showing deletion 5 bp of exon10 (c) RT-PCR analysis. Lane 1: Negative control (CN) in which no reverse transcriptase was added; lane 2: M, DNA marker $4 \times 174$ cut with Haelll; lane 3: Amplification product from c.1392 + 1G > C CFTR cDNA, and lane 4: Amplification product from c.1392 + 1G > T CFTR cDNA.

changing regulatory-sequence motifs of exonic splice enhancers, leading to increase skipping of exons 10 and 13 and lower amounts of normal transcripts [5].

This study aims at disclosing the new putative splicing polymorphism c.743 $+40 \mathrm{~A}>\mathrm{G}$ by hydride minigene assay beside its frequency in the general population. The clinical investigation and mutational data of patients, who had this polymorphism in the CFTR gene were identified. This finding has shown that this polymorphism is associated with CFTR-RD diseases. Moreover, in a family, this polymorphism is present in cis with c. 2562T > G and c.4389 G > A variants on the same allele in exons 15 and 27 respectively. The father has a severe pancreatic symptoms and his son has weak pancreatic inflammation with elevated sweat chloride levels in the absence of cystic fibrosis and any deleterious mutation. For this reason, we have chosen to study this nucleotide change. Here, we present epidemiological data in studied families. The c.743 $+40 \mathrm{~A}>\mathrm{G}$ polymorphism has been shown to be relatively frequent in French population (5\%) whereas, only $4 \%$ in the Spanich population [11]. Other previous studies screening for this variant in patients have been reported [6] [7] [9]. Moreover, single nucleotide substitutions may have a profound effect on the splicing efficiency inducing both exon inclusion and skipping. The changes in the splicing pattern were modulated by the composition of the polymorphic TG/T locus in intron 9 [18]. Thus, we have investigated the effect of c.743 + 40A > G using minigene constructs; the results revealed that this variant could not induce aberrant splicing of intron 6 . Therefore, this polymorphism could be considered as a non-pathogenic nucleotide change. However, we tested here only this polymorphism because it is the common point of characterized 16 patients. In the family presented, this polymorphism is always associated with c. $2562 \mathrm{~T}>\mathrm{G}$ and c.4389 G > A and we have no data concerning the effect of this complex allele on the CFTR 
splicing.

Nowadays, thousands of mutations are identified yearly. Although many directly affect protein expression, an increasing proportion of mutations is now believed to influence mRNA splicing. They mostly affect existing splice sites, but synonymous, non-synonymous or nonsense mutations can also create or disrupt splice sites or auxiliary cis-splicing sequences [19]. Furthermore, there are many mutations which identified as a putative splicing mutation not fully examined and confirmed its impact. Thus the use of an in vitro minigene assay could be extended to other mutations and polymorphisms believed to affect splicing.

\section{Conclusion}

This study concluded that the c.743 + 40A > G intronic variant has no clinical consequences on CFTR protein and could be classified as non-pathogenic polymorphism due to its effect on mRNA splicing. However, our conclusions have only been drawn from cell-culture experiments and transfection studies using in vitro minigene assay but in vivo assessment can validate the splicing outcome and provide justification for further experimental examination of patient samples, when available. These data provide a better characterization of this variant in CFTR-RD patients in France. In addition, the search for other associations with this polymorphism should be examined, especially with the complex alleles containing c.2562T > G and c.4389G > A, or TG12-7T. Further experiments are needed to determine the role of these complex alleles in the severity of CF disease.

\section{Acknowledgements}

This work was supported by Poitiers University and Hospital of Poitiers, France. We would like to thank James Habrioux for his assistance in preparation of the manuscript figures.

\section{References}

[1] http://www.genet.sickkids.on.ca/cftr

[2] Wang, D. and Sadée, W. (2006) Searching for Polymorphisms That Affect Gene Expression and mRNA Processing: Example ABCB1 (MDR1). Journal of the American Association of Pharmaceutical Scientists (AAPS), 8, 515-520.

[3] Nielsen, K.B., Sørensen, S., Cartegni, L., Corydon, T.J., Doktor, T.K., Schroeder, L.D., Reinert, L.S., Elpeleg, O., Krainer, A.R., Gregersen, N., Kjems, J. and Andresen, B.S. (2007) Seemingly Neutral Polymorphic Variants May Confer Immunity to Splicing-Inactivating Mutations: A Synonymous SNP in Exon 5 of MCAD Protects from Deleterious Mutations in a Flanking Exonic Splicing Enhancer. American Journal of Human Genetics, 80, 416-432. http://dx.doi.org/10.1086/511992

[4] Cuppens, H., Lin, W., Jaspers, M., Costes, B., Teng, H., Vankeerberghen, A., Jorissen, M., Droogmans, G., Reynaert, I., Goossens, M., Nilius, B. and Cassiman, J.J. (1998) Polyvariant Mutant Cystic Fibrosis Transmembrane Conductance Regulator Genes. The Polymorphic (TG)m Locus Explains the Partial Penetrance of the T5 Polymorphism as a Disease Mutation. Journal of Clinical Investigation, 101, 487-496. http://dx.doi.org/10.1172/JCI639

[5] Steiner, B., Truninger, K., Sanz J., Schalle, R.A. and Gallati, S. (2004) The Role of Common Single-Nucleotide Polymorphisms on Exon 9 and Exon 12 Skipping in Nonmutated CFTR alleles. Human Mutation, 24, 120-129. http://dx.doi.org/10.1002/humu.20064

[6] Chu, C.S., Trapnell, B.C., Curristin, S., Cutting, G.R. and Crystal, R.G. (1993) Genetic Basis of variable Exon 9 Skipping in Cystic Fibrosis Transmembrane Conductance Regulator mRNA. Nature Genetics, 3, 151-156. http://dx.doi.org/10.1038/ng0293-151

[7] Bombieri, C., Giorgi, S., Carles, S., De Cid, R., Belpinati, F., Tandoi, C., Pallares-Ruiz, C., Lazaro, C., Ciminelli, B.M., Romey, M.C., Casals, T., Pompei, F., Gandini, G., Claustres, M., Estivill, X., Pignatti, P.F. and Modiano, G. (2000) A New Approach for Identifying Non-Pathogenic Mutations: An Analysis of the Cystic Fibrosis Transmembrane Regulator Gene in Normal Individuals. Human Genetics, 106, 172-178. http://dx.doi.org/10.1007/s004390051025

[8] Luzardo, G., Aznarez, I., Crispino, B., Mimbacas, A., Martínez, L., Poggio, R., Zielenski, J., Tsui, L.C. and Cardoso, H. (2002) Cystic Fibrosis in Uruguay. Genetics and Molecular Research, 1, 32-38.

[9] Kolesar, P., Minarik, G., Baldovic, M., Ficek, A., Kovacs, L. and Kadasi, L. (2008) Mutation Analysis of the CFTR Gene in Slovac Cystic Fibrosis Patients by DHPLC and Sequencing: Identification of Four Novel Mutations. General Physiology and Biophysics, 27, 299-305.

[10] Loumia, O., Ferec, C., Mercier, B., Creff, J., Fercot, B., Denine, R. and Grangaud, J.P. (2008) CFTR Mutations in the Algerian Population. Journal of Cystic Fibrosis, 7, 54-59. http://dx.doi.org/10.1016/j.jcf.2007.04.004 
[11] Casals, T., Bassas, L., Egozcue, S., Ramos, M.D., Giménez, J., Segura, A., Garcia, F., Carrera, M., Larriba, A., Sarquella, J. and Estivill, X. (2000) Heterogeneity for Mutations in the CFTR Gene and Clinical Correlations in Patients with Congenital Absence of the vas Deferens. Human Reproduction, 15, 1476-1483. http://dx.doi.org/10.1093/humrep/15.7.1476

[12] Fanen, P., Ghanem, N., Vidaud, M., Besmond, C., Martin, J., Costes, B., Plassa, F. and Goossens, M. (1992) Molecular Characterization of Cystic Fibrosis: 16 Novel Mutations Identified by Analysis of the Whole Cystic Fibrosis Transmembrane Conductance Regulator (CFTR) Coding Regions and Splice Site Junctions. Genomics, 13, 770-776. http://dx.doi.org/10.1016/0888-7543(92)90152-I

[13] Le Marechal, C., Audrezet, MP., Quere, I., Raquenes, O., Langonne ,S. and Ferec, C. (2001) Complete and Rapid Scanning of the Cystic Fibrosis Transmembrane Conductance Regulator (CFTR) Gene by Denaturing High-Performance Liquid Chromatography (D-HPLC): Major Implications for Genetic Counselling. Human Genetics, 108, 290298. http://dx.doi.org/10.1007/s004390100490

[14] Pagani, F., Buratti, E., Stuani, C., Romano, M., Zuccato, E., Niksic, M., Giglio, L., Faraguna, D. and Baralle, F.E. (2000) Splicing Factors Induce Cystic Fibrosis Transmembrane Regulator Exon 9 Skipping through a Non Evolutionary Conserved Intronic Element. Journal of Biological Chemistry, 275, 21041-21047. http://dx.doi.org/10.1074/jbc.M910165199

[15] Faa, V., Incani, F., Meloni, C.D., Masala, M., Baffico, A.M., Seia, M., Cao, A. and Rosatelli, M.C. (2009) Characterization of a Disease-associated Mutation Affecting a Putative Splicing Regulatory Element in Intron 6b of the Cystic Fibrosis Transmembrane Conductance Regulator (CFTR) Gene. Journal of Biological Chemistry, 284, 30024-30031. http://dx.doi.org/10.1074/jbc.M109.032623

[16] Janssen, R., Kruit, A., Grutters, J.C., Ruven, H.J.T., van Moorsel, C.M.H. and van den Bosch, J.M.M. (2005) TIMP-3 Promoter Gene Polymorphisms in BFL. Thorax, 60, 974-975. http://dx.doi.org/10.1136/thx.2005.046581

[17] Davies, J., Alton, E. and Griesenbach, U. (2005) Cystic Fibrosis Modifier Genes. Journal of the Royal Society of Medicine, 45, 47-54.

[18] Pagani, F., Stuani, C., Tzetis, M., Kanavakis, E., Efthymiadou, A., Doudounakis, S., Casals, T. and Baralle, F.E. (2003) New Type of Disease Causing Mutations: The Example of the Composite Exonic Regulatory Elements of Splicing in CFTR Exon 12. Human Molecular Genetics, 12, 1111-1120. http://dx.doi.org/10.1093/hmg/ddg131

[19] Desmet, F.-O., Hamroun, D., Lalande, M., Collod-Béroud, G., Claustres, M. and Béroud, C. (2009) Human Splicing Finder: An Online Bioinformatics Tool to Predict Splicing Signals. Nucleic Acids Research, 37, 215-220. http://dx.doi.org/10.1093/nar/gkp215

\author{
Abbreviations \\ CF: Cystic Fibrosis \\ CFTR-RDs: CFTR-Related Disorders \\ DHPLC: Denaturing High Pressure Liquid phase Chromatography \\ DGGE: Denaturing Gradient Gel Electrophoresis \\ CBAVD: congenital bilateral absence of vas deferens \\ DB: disseminated bronchiectasis
}

